\title{
Two Turbulent Flow Regimes at the Inlet of a Rotating Pipe
}

\author{
Ferdinand-J. Cloos, Anna-L. Zimmermann, Peter F. Pelz*
}

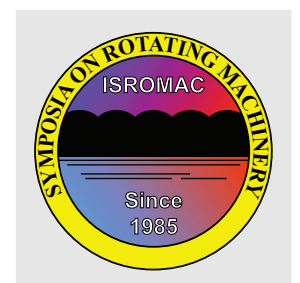

ISROMAC 2016

International

Symposium on

Transport

Phenomena and

Dynamics of

Rotating Machinery

Hawaii, Honolulu

April 10-15, 2016

\begin{abstract}
When a fluid enters a rotating circular pipe a swirl boundary layer with thickness of $\tilde{\delta}_{\mathrm{S}}$ appears at the wall and interacts with the axial momentum boundary layer with thickness of $\tilde{\delta}$. We investigate the turbulent flow applying Laser-Doppler-Anemometry to measure the circumferential velocity profile at the inlet of a rotating pipe. The measured swirl boundary layer thickness follows a power law taking Reynolds number and flow number into account. A critical combination of Reynolds number, flow number and axial position causes a transition of the swirl boundary layer development in the turbulent regime. At this critical combination, the swirl boundary layer thickness as well as the turbulence intensity increase and the latter yields a self-similarity. The circumferential velocity profile changes to a new presented self-similarity. A method is established to define the transition inlet length, when the transition appears and a stability map for two regimes is given.
\end{abstract}

Keywords

Boundary Layer — Transition — Rotating Pipe — Swirl Boundary Layer — Turbulent Flow Regimes

Chair of Fluid Systems, Technische Universität Darmstadt, Darmstadt, Germany

${ }^{*}$ Corresponding author: Peter.Pelz@fst.tu-darmstadt.de

\section{INTRODUCTION}

The interdependency of axial momentum and swirl becomes obvious at part load of a turbo machine. At part load below a critical flow number $\varphi:=\tilde{\bar{U}} /(\tilde{R} \tilde{\Omega})<\varphi_{\text {c }}$, with the average axial velocity $\tilde{\bar{U}}$ and circumferential velocity of the pipe $\tilde{R} \tilde{\Omega}$, the swirl causes separation $[1,2]$, the so called part load recirculation. We use a generic model of a turbo machine to investigate the evolution of the swirl and the impact of the swirl on the axial momentum balance; see Fig. 1. When an axial flow enters

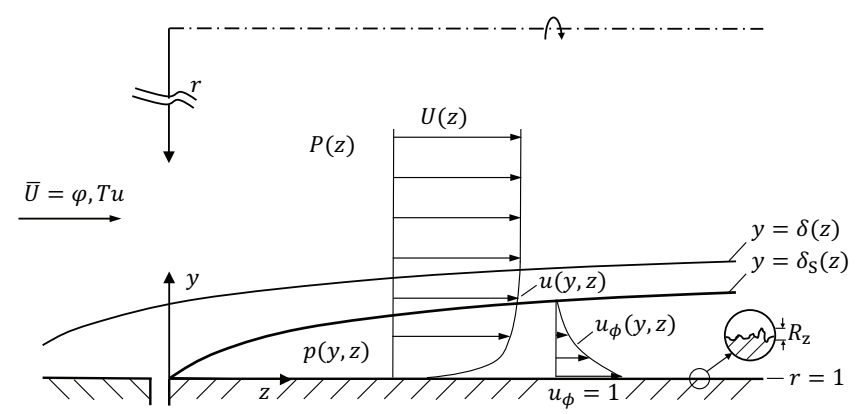

Figure 1. Inlet of a rotating pipe.

a pipe, an axial boundary layer with a thickness of $\tilde{\delta}$ appears and develops. By rotating the pipe, a second boundary layer in circumferential direction is produced by viscosity and develops, the so called swirl boundary layer having a thickness $\tilde{\delta}_{\mathrm{S}}$; see Fig. 1. Thus, close to the wall there is a region with a circumferential velocity component $\tilde{u}_{\phi}$. Due to centrifugal force, there is a non-negligible radial pressure distribution inside the swirl boundary layer for $\varphi \ll 1[1,2]$. Outside the swirl boundary layer, the flow is swirl-free. Outside the axial boundary layer, the flow is irrotational and the flow is accelerated due to the axial boundary layer growth. The evolution of both boundary layers depends on the Reynolds number $\operatorname{Re}:=2 \tilde{R}^{2} \tilde{\Omega} / \tilde{v}$, with the kinematic viscosity $\tilde{v}$, the flow number $\varphi$ and the averaged surface roughness $\tilde{R}_{z}$.

In this paper we present the experimentally investigated evolution of the swirl boundary layer and the circumferential velocity profile at high Reynolds numbers as well as high flow numbers in a rotating pipe. A critical combination of Reynolds number, flow number and axial position $(\operatorname{Re}, \varphi, z)_{\mathrm{t}}$ causes a transition of the swirl boundary layer development and a transformation of the circumferential velocity profile in the turbulent regime. We are looking for whether and where the transition appears by measuring the circumferential velocity component by Laser-Doppler-Anemometry (LDA). By doing so, we have two different inlet conditions, a thin (configuration I) and a fully developed (configuration II) turbulent axial boundary layer at the inlet of the rotating pipe. Throughout this investigation, we non-dimensionalize length with the pipe radius $\tilde{R}$ and velocities with the pipe circumferential velocity $\tilde{R} \tilde{\Omega}$. The superscript “ ” indicates dimensional symbols.

This paper is organized as follows: first, we give a literature review concerning the main investigations of flow in a rotating pipe. In the second section, the experimental methodology is described including the test-rig and the measurement uncertainty. The measurements done with this experimental set-up are presented in section 3 including the circumferential velocity profile, swirl boundary layer thickness and turbulence intensity. Section 4 serves to discuss the results concerning the actual state of the research, presented in section 1 . In 
this section, a method is presented to define the transition inlet length $z_{\mathrm{t}}$ and to generate a stability map for the two turbulent regimes of the swirl boundary layer. In the closure of this paper, we summarize our investigation by five major findings.

\section{LITERATURE REVIEW}

The flow in a rotating pipe has been much more investigated at the fully developed region than at the inlet region. At the fully developed region, the boundary layers reach the pipe centre and the velocity profiles are independent of the axial coordinate. The boundary layers reach the centre for $z>10^{2}$ for a turbulent flow, depending on Reynolds number and flow number [3]. Also the hydraulic losses of a turbulent flow decrease with decreasing flow number in a rotating pipe compared to a non-rotating pipe $[4,5]$. The centrifugal force damps the turbulence, stronger close to the wall than in the core, and stabilizes the flow [6]. At the inlet of a rotating pipe with a turbulent flow for $\varphi>1$, the turbulence is stimulated due to the sudden increase of the swirl for $z<20$. Further downstream the stabilization of the centrifugal force predominates $[7,8]$. The hydraulic losses are increased when a laminar flow enters a rotating pipe. Here, the swirl destabilizes the flow, stimulates the turbulence and thus, the transition to a turbulent flow occurs further upstream than in a non-rotating pipe [5].

An interaction of the swirl and the axial momentum is observed by a complex transformation of the axial velocity profile at the inlet of a rotating pipe. Due to turbulence damping, the axial velocity profile transforms continuously from a turbulent into a laminar profile in axial direction when a fully developed, turbulent flow enters the rotating pipe [9]. This effect is called "laminarization" and has been observed in many investigations, e.g. $[3,10,11]$. The "laminarized" profile reaches the fully developed region for a small flow number. For a higher flow number, the axial velocity profile is retransformed into the turbulent one [3]. An analytical approach of this transformation by Weigand and Beer [12] meets the experimental results qualitatively, but the influence of the swirl is overestimated.

The swirl boundary layer with its velocity profile defines the evolution of the swirl. The circumferential velocity profile is $u_{\phi}=r^{2}$, when a turbulent flow enters the rotating pipe $[11,13-15]$ and for a laminar flow it follows solid body rotation $u_{\phi}=r$ [11] at the fully developed region. Oberlack [16] derived the profiles by Lie group analysis. In the transition region, the circumferential velocity profile is between the parabolic and the linear profile [11]. When a thin laminar boundary layer enters a rotating pipe for $\varphi>0.71$, the circumferential velocity profile transforms and both boundary layers are thickened at the inlet of a rotating pipe [3]. There, for a smaller flow number with a thin or fully developed turbulent or a thin laminar axial boundary layer, the circumferential velocity profile follows $u_{\phi}=\left(1-y / \delta_{\mathrm{S}}\right)^{2}$ for an attached flow $[1,2,17]$. For the fully developed turbulent flow, the swirl boundary layer thickness follows

$$
\delta_{\mathrm{S}}=C \operatorname{Re}^{m_{1}} \varphi^{m_{2}} z^{m_{3}},
$$

with $C \approx 4.43, m_{1} \approx-0.45, m_{2} \approx-0.46$ and $m_{3} \approx 0.47$ until a transition occurs $[2,17]$, which is the main focus of this paper. For a thin axial boundary layer, the constant $C$ is approximately 4.64 and $m_{1} \approx-0.46, m_{2} \approx-0.49$ and $m_{3} \approx 0.44$ [2]. The swirl boundary layer becomes independent of the Reynolds number for a hydrauliclly rough wall but still depends on the flow number $[1,2]$. For a hydraulically rough wall, the circumferential velocity profile follows more or less $u_{\phi}=\left(1-y / \delta_{\mathrm{S}}\right)^{7}$ [2].

Equation 1 for a thin axial boundary layer is confirmed by our analytical approach [1,2]. By this approach, we use the integral method of boundary layer theory and generalize the von Kármán momentum equation taking the influence of swirl by a radial pressure distribution into account. A strong influence of the swirl on the axial momentum balance is observable for small flow numbers. The swirl causes flow separation for a small flow number and a measured stability map for part load recirculation is given $[1,2,17,18]$. When the flow separates, the circumferential velocity profile differs from the parabolic one $[1,2,17]$. Stratford's criteria [19] is applied to derive the critical flow number for incipient separation analytically. The results are validated by experiments [1]. For small Reynolds numbers, e.g. laminar flow, flow separation is investigated by Lavan and others in a rotating pipe [20-22].

\section{EXPERIMENTAL METHODOLOGY}

A test-rig is designed to measure the evolution of the swirl boundary layer and the circumferential velocity component in a rotating pipe applying 1D LDA. The swirl boundary layer thickness depends on the Reynolds number, the flow number, the axial position and the inlet condition. By doing so, two configurations with different inlet conditions are used, cf. $[1,2,17,18]$.

\subsection{Experimental Set-Up}

The air flow at ambient pressure is provided by a side channel blower, which increases pressure in a large plenum chamber, not shown in Fig. 2. In this plenum chamber, the air temperature is measured. The outlet of the plenum chamber is followed by a first flow straightener (cf. Fig. 2). Hence, pulsations are minimized. The volume flow is measured by an orifice plate and the flow is varied by changing the rotation speed of the blower. Thus, the axial velocity is controlled. The maximum axial velocity is $30 \mathrm{~m} / \mathrm{s}$, resulting in a Mach number smaller than 0.1 .

Downstream of the flow measurement an exchangeable section for different inlet conditions is implemented; see Fig 2. Both configurations include a second flow straightener. By configuration I, three turbulence screens are installed between a diffuser and the Börger-Nozzle [23]. By means of this nozzle, a thin axial boundary layer is generated due to flow acceleration, thus a bulk like velocity profile. Downstream 

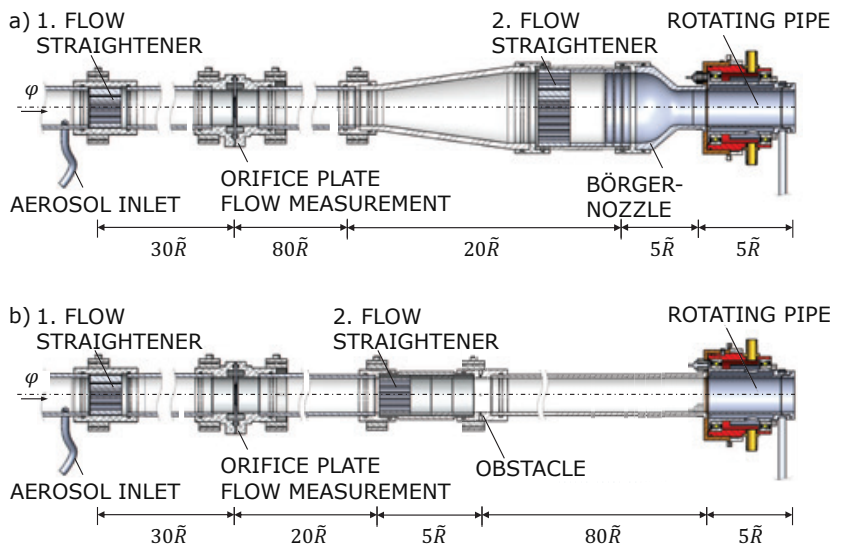

Figure 2. Experimental set-up with a) configuration I for a thin axial boundary layer and b) configuration II for a fully developed axial boundary layer.

of the Börger-Nozzle, the flow enters the rotating pipe as illustrated in Fig. 2. At the inlet of the rotating pipe, the axial boundary layer has a thickness of $\delta=8 \ldots 10 \%$ with a turbulent profile and $T u_{z}:=\tilde{u}_{\mathrm{rms}}^{\prime} / \tilde{U}_{\max } \approx 1 \ldots 2 \%$ outside the boundary layer for $\tilde{\bar{U}} \geq 8 \mathrm{~m} / \mathrm{s}$. Inside the boundary layer, the turbulence intensity increase approximately to $13 \%$. For configuration II, an obstacle is installed, see Fig. 2b, to generate a fully developed, turbulent boundary layer. At the inlet of the rotating pipe, the axial boundary layer thickness is 1 for $\tilde{\bar{U}} \geq 2 \mathrm{~m} / \mathrm{s}$. The turbulence intensity is approximately $4 \%$ in the pipe centre and approximately $12 \%$ closer to the wall. The inlet conditions of both configurations are measured by a hot wire anemometer.

Between the non-rotating and rotating pipe there is an axial gap of $4 \%_{0} \tilde{R}$. The gap is sealed by sealed ball bearings. The rotating pipe radius $\tilde{R}$ is $25 \mathrm{~mm}$, its length is $5 \tilde{R}$ and has a relative surface roughness of $0.04 \% \tilde{R}$. The rotating pipe is driven by a belt. The maximum rotational speed is $\tilde{\Omega}=1308 \mathrm{rad} / \mathrm{s}$, yielding a maximum Reynolds number of $\log (R e)=5.1$. Hence, Reynolds number and flow number are independent of each other. The outlet of the pipe is a free jet. The advantage of this design is a convenient accessibility from downstream to the flow field within the rotating pipe.

This accessibility is used to measure the circumferential velocity component by a 1D LDA with frequency shift and a wave length of $514.5 \mathrm{~nm}$. The probe has a focus of $310 \mathrm{~mm}$ and is located downstream of the rotating pipe. The measurement volume has a length of $<1.6 \% \tilde{R}$, a diameter of $<2 \% \tilde{R}$ and is moveable with a two dimensional plane by using a traverse table. An aerosol of silicon oil as tracer particles is added to the air to enable LDA measurements; see Fig. 2. The LDA and the experimental set-up is described in more detail by $[1,2,17]$.

\subsection{Measurement Uncertainty}

For the Reynolds number the temperature in the plenum chamber with a systematic measuring error of $\pm 0.5^{\circ} \mathrm{C}$ and the rotational speed with a systematic measuring error of $\pm 5 \mathrm{~Hz}$ is measured. Sutherland's law [24] and the equation of state for a perfect gas yields the kinematic viscosity with a systematic measuring error of $\Delta v_{\mathrm{sys}} / v= \pm 2 \%$. Thus, the maximum systematic measuring error of Reynolds number is less than

$$
\frac{\Delta R e_{\mathrm{sys}}}{\operatorname{Re}}=\left|\frac{\Delta \tilde{\Omega}_{\mathrm{sys}}}{\tilde{\Omega}}\right|+\left|\frac{\Delta \tilde{v}_{\mathrm{sys}}}{\tilde{v}}\right| \leq \pm 4 \%
$$

for $\log (R e)=4.1$.

The orifice plate is designed in agreement with ISO 5167-2:2003 [25]. Evaluating the systematic measuring error of flow measurement, pressure losses by the orifice plate are assumed to be proportional to the square of the velocity. The resistant coefficient of the orifice plate has a systematic measuring error of $\pm 0.5 \%$ [25], but we assume $\pm 1 \%$ for a conservative evaluation, yielding $\Delta \tilde{\bar{U}}_{\text {sys }} / \tilde{\bar{U}} \leq \pm 3.5 \%$. Hence, the systematic measuring error of flow number is less than

$$
\frac{\Delta \varphi_{\text {sys }}}{\varphi}=\left|\frac{\Delta \tilde{\Omega}_{\text {sys }}}{\tilde{\Omega}}\right|+\left|\frac{\Delta \tilde{\bar{U}}_{\text {sys }}}{\tilde{\tilde{U}}}\right| \leq \pm 5.5 \%
$$

for $\varphi=0.35$ and $\log (R e)=4.1$. The systematic measuring errors of the Reynolds number and the flow number decrease with increasing Reynolds number and flow number, respectively. Both quantities are measured over $10 \mathrm{~s}$ including 200 data points. The precision error of Reynolds number and flow number is calculated with

$$
\Delta x_{\text {stat }}= \pm \frac{t(N)}{\sqrt{N}} s(x)
$$

where $t$ denotes the Student's factor, $N$ the sample number and $s$ the standard deviation. For $N=200$, the Student's factor is 1.96 for a $95 \%$ confidence interval.

The positioning of the LDA measuring volume has a systematic measuring error of $\Delta \tilde{z}_{\text {sys }} \approx \pm 8 \% \tilde{R}$ and $\Delta \tilde{y}_{\text {sys }} \approx \pm 4 \% 0 \tilde{R}$. The repetitive accuracy in both directions of the traverse table is $0.8 \% \tilde{R}$. One LDA data point is measured over more than $30 \mathrm{~s}$ including more than 1000 bursts. The transit time of each passing tracer particle is used to weight the measured velocity. In the following, all presented data points include the total measuring error by errors bars in accordance with

$$
\Delta x=\sqrt{\left(\Delta x_{\mathrm{sys}}\right)^{2}+\left(\Delta x_{\mathrm{stat}}\right)^{2}} .
$$

Usually, the error bars are smaller than the marker, thus they are not visible and the precision error of $\tilde{u}_{\phi, \text { rms }}^{\prime}$ is unknown.

\section{RESULTS}

In this section we vary the Reynolds number and the flow number to investigate the evolution of the swirl. First, the swirl velocity distribution, second, the swirl boundary layer thickness and third, the turbulence intensity $T u:=\tilde{u}_{\phi, \mathrm{rms}}^{\prime} /(\tilde{R} \tilde{\Omega})$ is presented. 

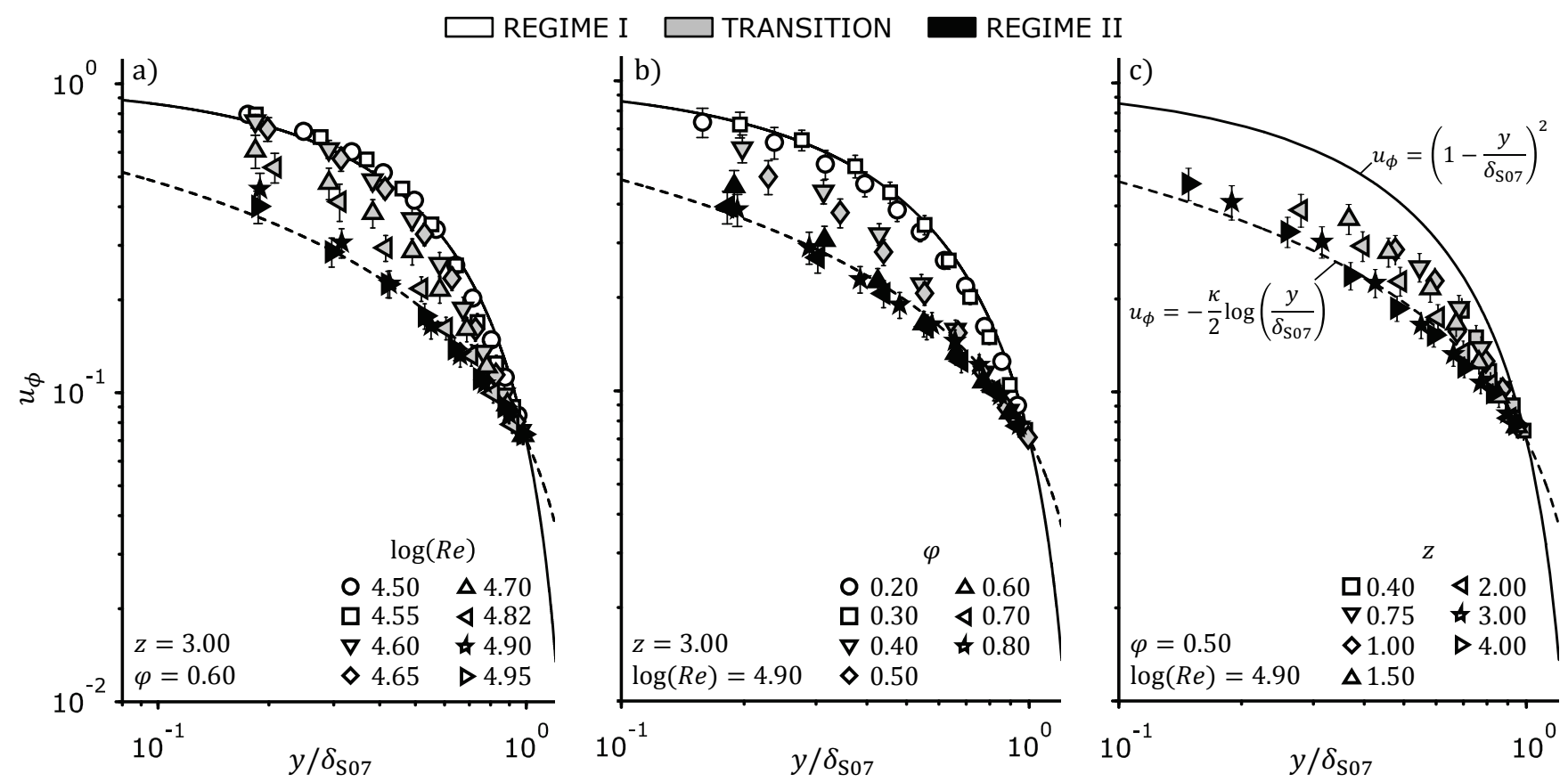

Figure 3. Circumferential velocity distribution for a) various Reynolds number, b) various flow number and c) various axial position (configuration I).

\subsection{Circumferential Velocity Profile}

The circumferential velocity profile for varying Reynolds number, varying flow number and varying axial position is shown in Fig. 3. The velocity profile is scaled with the swirl boundary thickness $\delta_{\mathrm{S} 07}$, where $u_{\phi}\left(\delta_{\mathrm{S} 07}, z\right)=0.07$.

Already Fig. 3 indicates our findings: there are two turbulent flow regimes I and II. First, below a critical parameter combination $(\operatorname{Re}, \varphi, z)_{\mathrm{t}}$ the circumferential velocity profile is the well known one $[1,2,11,13-16]$

$$
u_{\phi}=\left(1-\frac{y}{\delta_{\mathrm{S} 07}}\right)^{2} \text { for } \operatorname{Re}<\operatorname{Re}_{\mathrm{t}}(\varphi),
$$

indicated by the white markers in Fig. 3 and the solid line. We observe a transition from regime I to II being well depictured by Fig. 3a to $3 \mathrm{c}$. The black markers coincide with the dashed line given by the logarithmic law

$$
u_{\phi}=-\frac{\kappa}{2} \log \left(\frac{y}{\delta_{\mathrm{S} 07}}+0.007\right)+0.06 \text { for } \operatorname{Re}>\operatorname{Re}_{\mathrm{t}}(\varphi)
$$

with $\kappa=0.4$. The grey markers illustrate the transition from regime I to regime II. Hence, there is no sharp transition surface $(\operatorname{Re}, \varphi, z)_{\mathrm{t}}$. As will be seen in the following section the transition surface $(\operatorname{Re}, \varphi, z)$ t reduces to a transition line $\operatorname{Re}_{\mathrm{t}}(\varphi)$.

\subsection{Swirl Boundary Layer Thickness}

Figure 4 illustrates the swirl boundary layer thickness for various flow number and Reynolds number at different axial positions, respectively. According the circumferential velocity profile transformation, the swirl boundary layer thickens when the critical combination is reached (cf. black markers in Fig. 4). The dependency of $\delta_{\mathrm{S}}$ on the axial coordinate, flow number and Reynolds number changes.

\subsection{Turbulence Intensity}

In Fig. 5 the turbulence intensity depending on flow number and Reynolds number at different axial positions is shown. The turbulence intensity increases for the critical parameter combination until it yields a self-similarity as the black markers illustrate.

\section{DISCUSSION}

The circumferential velocity profile and the turbulence intensity for regime II is similar to the case of a hydraulically rough wall [2]. For a rough surface, the flow is independent of Reynolds number [1,2], being not observed in our case, e.g. regime II.

One might be think of a flow separation causing the observed transition from regime I to regime II. But this is not the case. Our previous results $[1,2,17,18]$ indicate flow separation (so called part load recirculation) for $\varphi_{c}=0.1 \ldots 0.2$. Here, we are well above this critical range.

Facciolo et al. [26] presented a calculation of the circumferential velocity profile at the fully developed region with the angular momentum balance. Thereby, the Reynolds stress $\overline{u^{\prime} u_{\phi}^{\prime}}$ transforms the profile from the linear to the parabolic profile. Thus, the increase of the turbulence intensity could justify the profile transformation at the developing inlet region.

According to the turbulent axial boundary layer at the inlet of the rotating pipe, this could not be a transition to a turbulent 


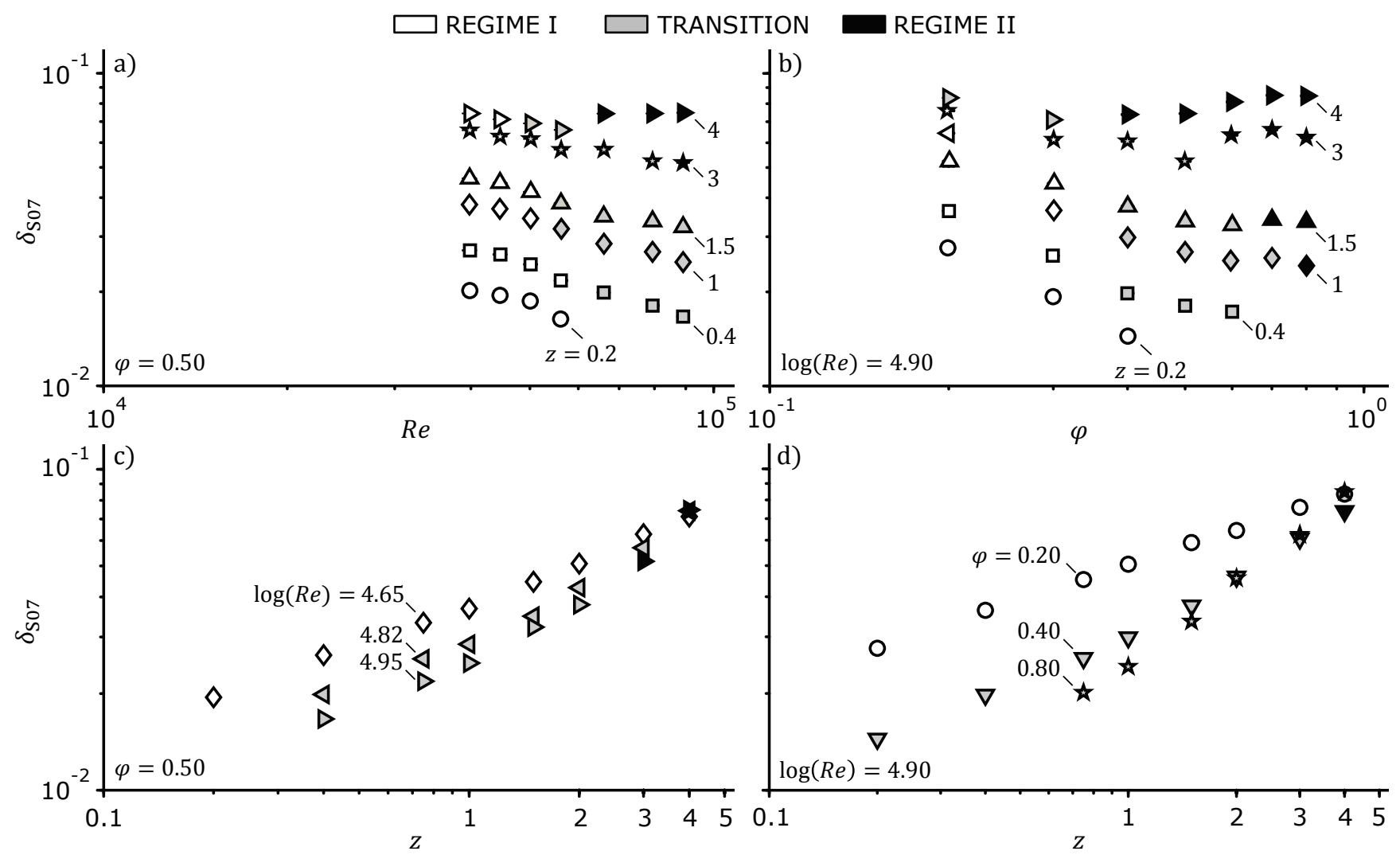

Figure 4. Swirl boundary layer thickness for a) and c) constant flow number and b) and d) constant Reynolds number (configuration I).

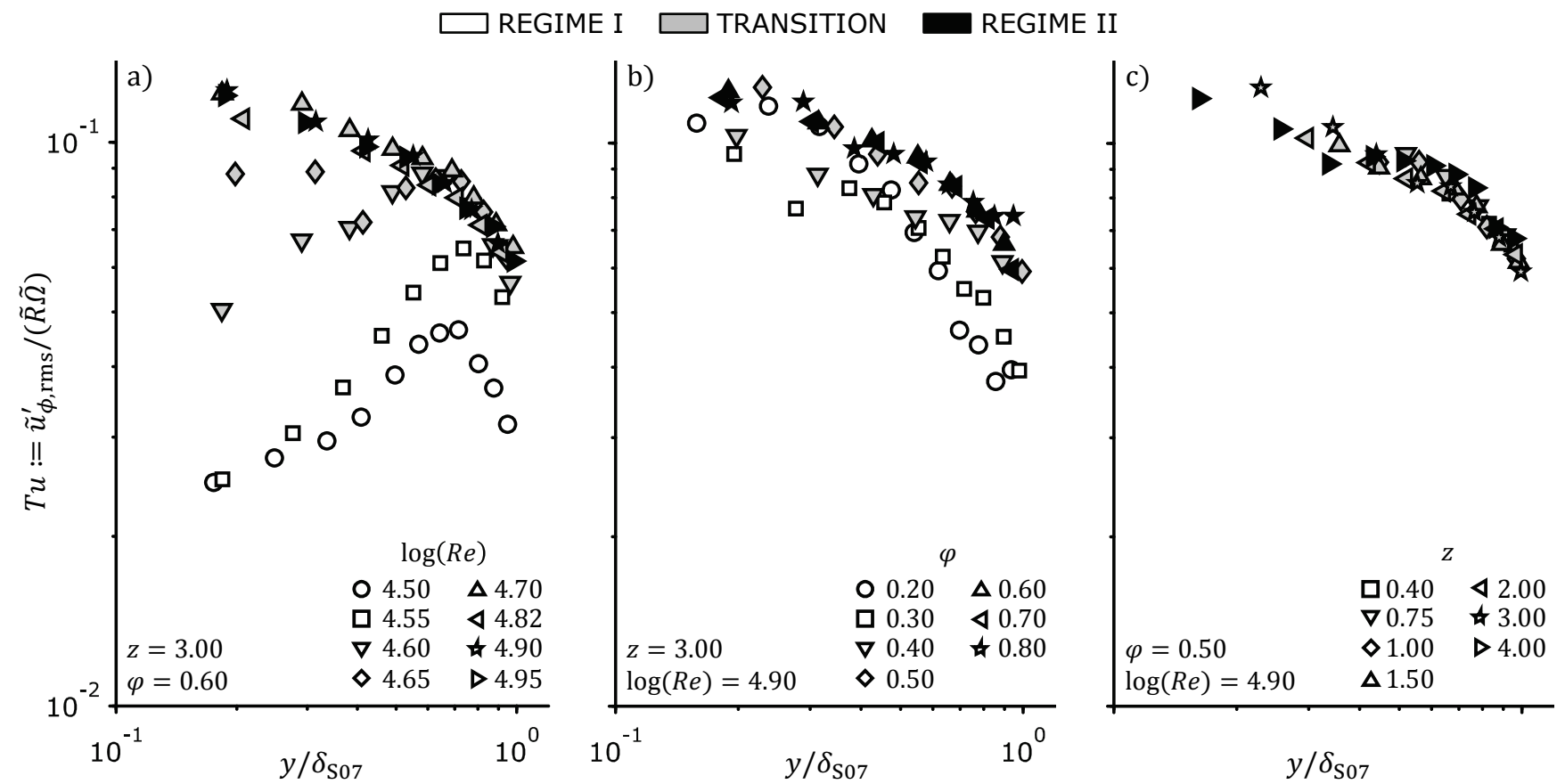

Figure 5. Turbulence intensity for a) various Reynolds number, b) various flow number and c) various axial position (configuration I). 
flow. Among other things, this is justified by the self-similar circumferential profile for a turbulent flow which follows $u_{\phi}=\left(1-y / \delta_{\mathrm{S}}\right)^{2}$ below the critical parameter combination. The swirl stimulates the turbulence when a laminar flow enters a rotating pipe [5], but here, a turbulent flow enters the rotating pipe and we do not observe a damping of the turbulence intensity. At the inlet region, the stimulation of the turbulence predominates $[7,8]$. To summarize, our findings indicate an up to now unknown transition from one turbulent regime to another in a rotating pipe.

We apply a self-developed method to indicate the transition. The method bases on a comparison between the measured circumferential and a comparative velocity profile. For regime I, the swirl velocity profile follows the well-known parabolic profile (Eq. 6). The velocity profile changes to a suggested logarithmic profile (Eq. 7) for regime II of the swirl boundary layer. We are using this fact as a main indicator to classify the turbulent flow. By doing so, the procedure starts with a quantitative comparison of each measurement point of the respective velocity profile. Hence, the velocity profile itself can be attributed to a regime. The decision about the corresponding regime is deduced from the majority of the measured points which are categorized to one of the two selfsimilarities, e.g. regime I or regime II. If the majority of the measurement points can be attributed to neither regime I nor regime II, the swirl boundary layer is rated among the so called transition area. Due to uncertainty in the measured velocity profiles, we take a particular tolerance range into account to classify each swirl velocity profile. For both cases, regime I and II, we define a range of $\pm 5 \%$ to the given comparative velocity profiles (cf. Fig. 6).

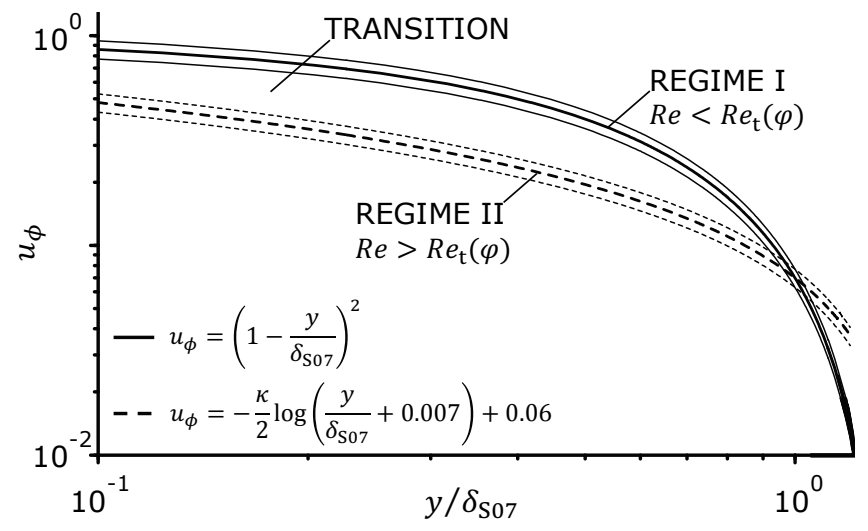

Figure 6. Method to indicate the regime I and regime II of the swirl boundary layer.

With the help of the method we are now set to quantify the so far unknown transition surface $(\operatorname{Re}, \varphi, z)$ t. Figure 7 indicates the transition inlet length $z_{\mathrm{t}}$ to be independent of Reynolds number yielding $z_{\mathrm{t}}=z_{\mathrm{t}}(\varphi)$. Hence, together with this result the transition is solely described by a plane stability map $(\operatorname{Re}, \varphi)_{\mathrm{t}}$ and the transition is given by one single curve $\operatorname{Re}_{\mathrm{t}}(\varphi)$ as sketched in Fig. 8.

When a fully developed, turbulent flow enters the rotating pipe, there is also a transition of the swirl boundary layer

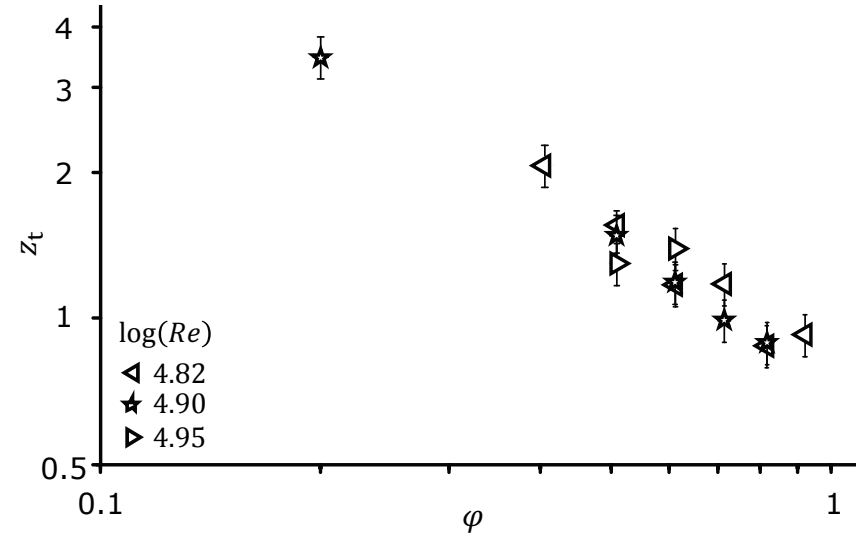

Figure 7. Transition inlet length of the swirl boundary layer (configuration I).

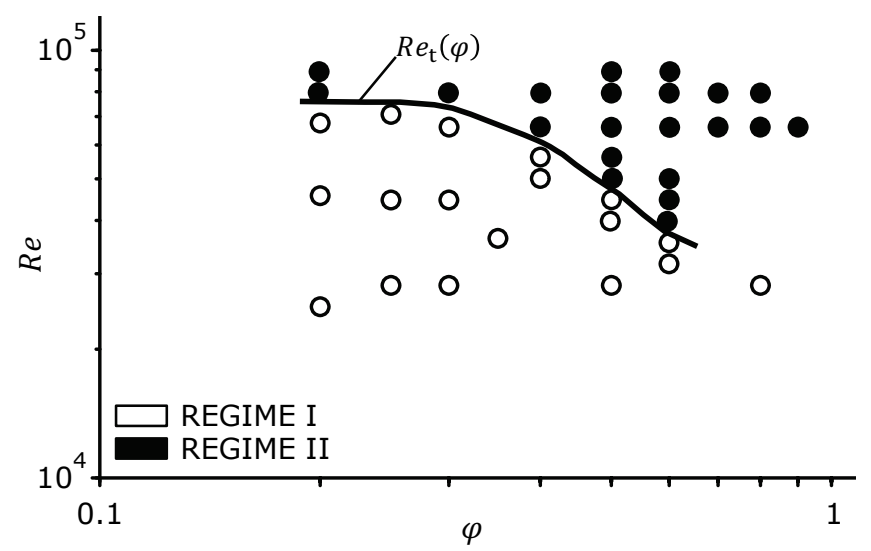

Figure 8. Stability map for regime I and regime II of the swirl boundary layer (configuration I).

development; see Fig. 9. The investigation of the transition for a fully developed flow is published by [27]. Thus, the transition occurs for both configurations.

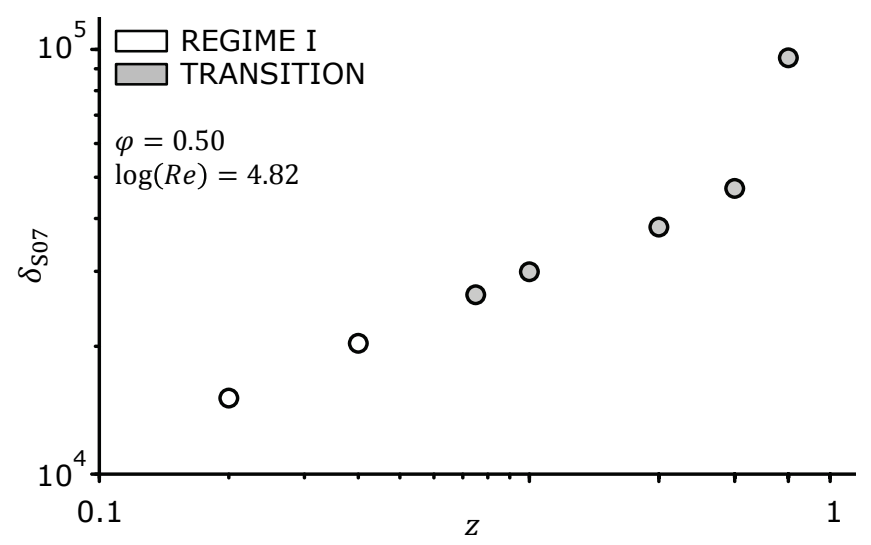

Figure 9. Instability of the swirl boundary layer (configuration II). 


\section{CONCLUSION}

In this paper, we analysed the swirl evolution at the inlet of a rotating pipe with a length of $5 \tilde{R}$ at high Reynolds numbers and high flow numbers. The swirl distribution and evolution follows the known regularity for $(\operatorname{Re}, \varphi, z)<(\operatorname{Re}, \varphi, z)_{\mathrm{t}}$, regime I. For $(\operatorname{Re}, \varphi, z)>(\operatorname{Re}, \varphi, z)$, we observed a second transformation in the turbulent regime with five major findings:

1. For regime II, the circumferential velocity profile is $u_{\phi}=-\frac{\kappa}{2} \log \left(\frac{y}{\delta_{\mathrm{S} 07}}+0.007\right)+0.06$.

2. The swirl boundary layer thickens and the dependency on the axial coordinate, the flow number and the Reynolds number changes.

3. The turbulence intensity increase and yields a new selfsimilarity.

4. Where and whether the regime II appears is given by the transition inlet length, cf. Fig. 7, and the stability map Fig. 8 .

5. The transformation into regime II is observable when a fully developed and a thin turbulent axial boundary layer enters the rotating pipe.

The investigation of the axial boundary layer is necessary, to analyse the transformation completely. Thus, the axial boundary layer is in the next research focus.

\section{ACKNOWLEDGMENTS}

The authors would like to thank the German Research Foundation DFG for funding this research within PE 1573/5-1.

\section{REFERENCES}

[1] F.-J. Cloos, D. Stapp, and P.F. Pelz. Swirl boundary layer and flow separation at the inlet of a rotating pipe. submitted to J. Fluid Mech., 2015.

[2] D. Stapp. Experimentelle und analytische Untersuchung zur Drallgrenzschicht. Forschungsberichte zur Fluidsystemtechnik, 2015.

[3] K. Nishibori, K. Kikuyama, and M. Murakami. Laminarization of turbulent flow in the inlet region of an axially rotating pipe. Bulletin of JSME, 30:255-262, 1987.

[4] F. Levy. Strömungserscheinungen in rotierenden Rohren. PhD thesis, Technischen Hochschule München, 1927.

[5] A. White. Flow of a fluid in an axially rotating pipe. J. Mech. Engng. Sc., 6:47-52, 1964.

[6] A.I. Borisenko, O.N. Kostikov, and V.I. Chumachenko. Experimental study of turbulent flow in a rotating channel. J. Engng. Phys. and Thermophys, 24:770-773, 1973.

[7] H. Nagib, Z. Lavan, A.A. Fejer, and L.J. Wolf. Experimental study of turbulent flow in a rotating channel. J. Engng. Fluids, 24:770-773, 1973.

[8] L. Bissonnette and G. Mellor. Experiments on the behavior of an axisymmetric turbulent boundary layer with a sudden circumferential strain. J. Fluid Mech., 63:369413, 1974.

[9] K. Kikuyama, M. Murakami, K. Nishibori, and K. Maeda. Flow in an axially rotating pipe. a calculation of flow in the saturated region. Bulletin of JSME, 26:506-513, 1983.

[10] B. Weigand and H. Beer. On the universaility of the velocity profiles of a turbulent flow in an axially rotating pipe. Appl. Sc. Research, 52:115-132, 1994.

[11] S. Imao, M. Itohi, and T. Harada. Turbulent characteristics of the flow in an axially rotating pipe. Int. J. of heat and fluid flow, 17(5):444-451, 1996.

[12] B. Weigand and H. Beer. Fluid flow and heat transfer in an axially rotating pipe: the rotational entrance. In Proceedings of the 3rd ISROMAC, pages 325-340, 1992.

[13] M. Murakami and K. Kikuyama. Turbulent flow in axially rotating pipes. J. Fluids Engng., 102:97-103, 1980.

[14] K. Kikuyama, M. Murakami, and K. Nishibori. Developement of three-dimensional turbulent boundary layer in an axially rotating pipe. J. Fluids Engng., 105:154160, 1983.

[15] G Reich. Strömung und Wärmeübertragung in einem axial rotierenden Rohr. $\mathrm{PhD}$ thesis, Technischen Hochschule Darmstadt, 1988.

[16] M. Oberlack. Similarity in non-rotating and rotating turbulent pipe flows. J. Fluid Mech., 379:1-22, 1999.

[17] D. Stapp and P.F. Pelz. Evolution of swirl boundary layer and wall stall at part load - a generic experiment. In Proceedings of ASME Turbo Expo GT2014-26235, 2014.

[18] D. Stapp, P.F. Pelz, and J.M. Loens. On part load recirculation of pumps and fans - a generic study. In Proceedings of the 6th International Conference On Pumps an Fans with Compressors and Wind Turbines, page 022003 , 2013.

[19] B.S. Stratford. The prediction of separation of the turbulent boundary layer. J. Fluid Mech., 5:1-16, 1959.

[20] Z. Lavan, H. Nielsen, and A.A. Fejer. Separation and flow reversal in swirling flows in circular ducts. Phys. Fluids, 12:1747-1757, 1969.

[21] S. Imao, Q. Zhang, and Y. Yamada. The laminar flow in the developing region of a rotating pipe. Bulletin of JSME, 32:317-323, 1989.

[22] C. Crane and D. Burley. Numerical studies of laminar flow in ducts and pipes. J. Comp. and appl. Mathe., 2:95-111, 1976.

[23] G.-G. Börger. Optimierung von Windkanaldüsen für den Unterschallbereich. $\mathrm{PhD}$ thesis, Ruhr-Universität Bochum, 1973.

[24] W. Sutherland. The viscosity of gases and molecular force. The London, Edinburgh, and Dublin Philosophical Magazine and J. of Sc., 36(223):507-531, 1893. 
[25] ISO 5167-2:2003 Measurement of fluid flow by means of pressure differential devices inserted in circular crosssection conduits running full - Part 2: Orifice plates, 2003.

[26] L. Facciolo, N. Tillmark, A. Talamelli, and P.H. Alfredsson. A study of swirling turbulent pipe and jet flows. Phys. Fluids, 19:1-18, 2007.

[27] A.-L. Zimmermann. Instabilitäten im Eintrittsbereich eines rotierenden Rohres. Master's thesis, Technische Universität Darmstadt, 2015. 